Kompass

Onkologie

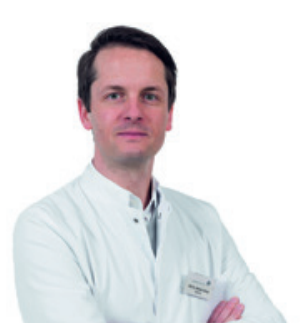

Rainer Claus

II. Medizinische Klinik, Universitätsklinikum Augsburg, Augsburg, Deutschland

\title{
CLL: Einblicke in aktuelle Entwicklungen der Behandlung
}

Die Therapie der chronischen lymphatischen Leukämie (CLL) hat in den letzten Jahren einen außerordentlich tiefgreifenden Wandel erfahren. Die Gründe hierfür liegen insbesondere in der Verfügbarkeit neuer molekularbiologischer Methoden, die in den letzten 20 Jahren zunehmend tieferes Verständnis der wesentlichen molekularen Pathomechanismen ermöglicht haben. Damit verbunden ist nicht nur die Entwicklung neuer prognostischer Marker, die eine präzisere Risikostratifizierung der CLLPatienten erlauben, sondern vor allem auch ein Fortschritt hin zu neuen zielgerichteten Therapieansätzen. Von der europäischen Zulassungsbehörde (EMA) wurden seit 2010 allein sieben neue zielgerichtete Substanzen für die Therapie der CLL in unterschiedlichen klinischen Situationen zugelassen. Das Wissen um die zentrale pathomechanistische Rolle von gesteigerter B-Zell-Rezeptor-Signaltransduktion und insuffizienter Apoptosevermittlung hat dazu geführt, dass genau diese Mechanismen als Achillesfersen der CLL identifiziert wurden, an denen die wirkungsvollsten Therapieoptionen, BTK (Bruton-Tyrosinkinase)-Inhibitoren und BH3-Mimetika, angreifen. Darüber hinaus befinden sich neue Substanzen und neue Generationen bekannter Substanzklassen in klinischer Testung. Dank höherer Wirksamkeit und besserer Verträglichkeitsprofile ist es möglich, das
Ansprechen und die Prognose gegenüber klassischen immunchemotherapeutischen Ansätzen grundlegend zu verbessern. Dies hat in der leitliniengerechten Behandlung von CLL-Patienten bereits in den letzten Jahren zu einem therapeutischen Paradigmenwechsel geführt, der damit einhergeht, dass die neuen zielgerichteten Wirkstoffe bisherige Chemoimmuntherapieregime weitestgehend ablösen.

Mit der Entwicklung effektiver, zielgerichteter Therapieoptionen hat sich auch eine Verschiebung des Therapieziels ergeben. Während Symptomkontrolle vor Jahren noch im Vordergrund der CLL-Therapie stand, spielt das Erreichen von tiefen Remissionen und eine bestmögliche Verlängerung von progressionsfreier und auch therapiefreier Zeit eine zunehmend wichtigere Rolle. Ultratiefes Ansprechen, im Sinne von Negativität für den Nachweis minimaler Resterkrankung (minimal residual disease, MRD), besitzt bei der CLL prognostische Bedeutung und scheint insbesondere für das Absetzen von Therapie bzw. die Therapiesteuerung eine wesentliche Bedeutung zu besitzen. Ob jedoch MRD-Negativität für jeden Patienten und jedes Therapieregime das relevanteste Therapieziel darstellt, ist bisher nicht klar.

Eine zentrale offene Frage ist aktuell, wann und bei welchem CLL-Patienten zeitlich limitierte Therapieregime (z.B. bei Veneto- 
clax-basierten Therapiekombinationen) oder kontinuierliche Therapie (z.B. bei BTKInhibitoren) sinnvoll sind. Gibt es Subgruppen von CLL-Patienten, die z.B. aufgrund der Proliferationskinetik des CLL-Klons oder anderer CLL-spezifischer Charakteristika dauerhaft therapiert werden sollten? Ist das Erreichen von MRD-Negativität eine Voraussetzung, um Dauertherapie gegebenenfalls doch zeitlich limitieren zu können? Viele dieser Fragen sind bisher nicht abschließend geklärt und stellen den Gegenstand aktueller Studiengenerationen dar.

Bei der Vielfalt der verfügbaren Therapieoptionen und dem Wissen um Resistenzentwicklung auch gegenüber den zielgerichteten Substanzen besteht eine Herausforderung darin, wie Therapiesequenzen sinn- und wirkungsvoll gestaltet werden können. Einen Schritt weiter geht die Frage, wie wir die verfügbaren Substanzen möglichst synergistisch kombinieren können. Die zugrunde liegende Idee hierfür ist, dass durch zweckmäßige Kombinationen sowohl größere Effektivität als auch die Möglichkeit zeitlich begrenzter Behandlung erreicht werden, bei der ein Patient die Be- handlung basierend auf der Qualität seines Ansprechens (z.B. MRD-Negativität) beenden kann.

Auch wenn die CLL weiterhin als nicht heilbare Erkrankung gilt, so stellt sich die Frage, ob durch wirkungsvolle Kombinationen der neuen Substanzen in Kombination mit MRD-gesteuerten Absetzstrategien sehr langanhaltende Remissionen und ggf. funktionelle Heilung erreicht werden können.

Abschließend sei noch auf die Möglichkeit der CAR (chimeric antigen receptor)-T-ZellTherapie, also modifizierte T-Zellen mit AntiCD19-CARs, hingewiesen. Obwohl die erste publizierte Studie zur CAR-T-Zell-Therapie für CLL veröffentlicht wurde, gibt es bisher noch keine zugelassene CAR-T-Therapieoption für diese Erkrankung. Ein Grund hierfür ist unter anderem, dass die CLL durch umfassende Immunsuppression, etwa des T-Zell-Kompartiments, gekennzeichnet ist, die die Wirksamkeit der CAR-T-Zellen beeinträchtigt. Aktuelle Studien wie die TRANSCEND-CLL004-Studie mit Lisocabtagene maraleucel zeigen im rezidivierten/refraktären Setting mit oder ohne BTK-Inhibitor, dass auch hier durch sinnvolle immun- modulierende Kombinationen wesentliche Fortschritte erzielt werden können.

Immundefizienz bei CLL-Patienten stellt auch im Sinne supportiver Therapiemaßnahmen gerade in der aktuellen Situation der SARS-CoV-2-Pandemie ein Problem im alltäglichen Management von CLL-Patienten dar. Wie sieht das COVID-19-Risikoprofil von CLL-Patienten aus? Welche Rolle spielt dabei B-Zell-depletierende CLL-Therapie mit den neuen zielgerichteten Substanzen? In dieser Ausgabe des Kompass Onkologie sollen einige der angesprochenen Fragen anhand aktueller Studiendaten näher erörtert und diskutiert werden. Wir befinden uns seit einigen Jahren in einer sehr spannenden und dynamischen Phase der CLLBehandlung und möchten Ihnen mit dem vorliegenden Beitrag einen Einblick in aktuelle Entwicklungen und die entsprechende Evidenzbasis geben.

Mit kollegialen Grüßen

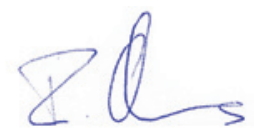

PD Dr. Rainer Claus 